\title{
Papulopustular skin lesions are seen more frequently in patients with Behçet's syndrome who have arthritis: a controlled and masked study
}

\author{
E Diri, C Mat, V Hamuryudan, S Yurdakul, N Hizlı, H Yazıcı
}

\begin{abstract}
Department of Internal Medicine, Rheumatic Diseases Division, University of Texas Southwestern Medical Centre, Dallas, Texas, USA E Diri
\end{abstract}

Department of Dermatology, Cerrahpasa Medical Faculty, University of Istanbul, Istanbul, Turkey C Mat

Department of Internal Medicine, Division of

Rheumatology, Cerrahpasa Medical Faculty, University of Istanbul, Istanbul, Turkey

V Hamuryudan

S Yurdakul

N Hizlı

H Yazıc1

Correspondence to: Professor H Yazıc1, Safa sok $17 / 7$, Kadikoy, Istanbul 81310, Turkey

hyazici@attglobal.net

Accepted 2 July 2001

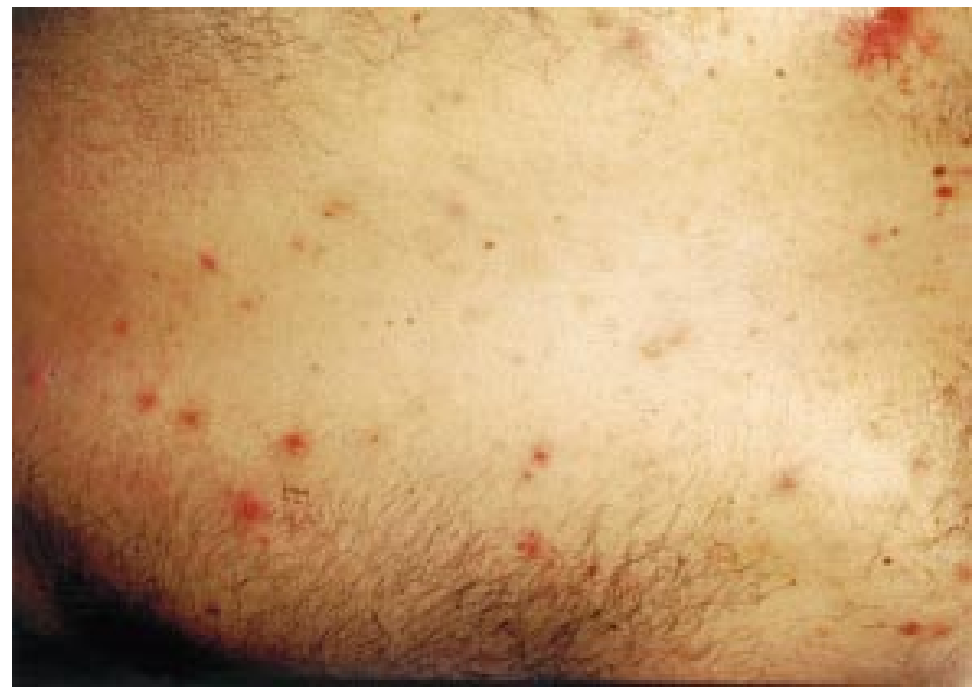

Figure 1 Acneiform skin lesions on the back of a patient with Behçet's syndrome.

\begin{abstract}
Objective-To determine the prevalence of acneiform skin lesions (comedones, papules, and pustules) in patients with Behçet's syndrome (BS) with arthritis. Methods-Study groups included 44 patients with BS with arthritis (32 men, 12 women, mean (SD) age 37.8 (8.9)), 42 patients with BS without arthritis (31 men, 11 women, mean age 35.5 (6.4)), 21 patients with active rheumatoid arthritis (five men, 16 women, mean age 48.8 (14)), and 33 healthy volunteers $(28$ men, five women, mean age $40.1(8.1)$ ). All probands and controls were examined by a rheumatologist and a dermatologist, in a prospective and masked protocol. An ophthalmological evaluation was performed if necessary. Skin lesions, including comedones, papules, and pustules, were counted and scored as 0 : absent, $1: 1-5,2$ : 6-10, 3: 11-15, 4: 16-20, and 5: >20.

Results-Although there was no significant difference between the four groups in the prevalence of comedones, the number of papules and pustules was significantly higher in patients with BS with arthritis $(p=0.0037$ for papules and $p<0.0001$ for pustules) than in the remaining three groups.

Conclusion-Acneiform skin lesions (papules and pustules) seem to be more frequent in patients with BS with arthritis. This suggest that the arthritis seen in BS may possibly be related to acne associated arthritis. (Ann Rheum Dis 2001;60:1074-1076)
\end{abstract}

\section{:} to another room to sit in a chair with their hands and feet covered. Patients with RA with deformities that could not be camouflaged with ease were excluded. An experienced dermatologist, with no prior knowledge of the identity of the people in the study group, counted the skin lesions, including comedones, papules, and pustules, on the face, the back, and the chest. For patients with BS the HLA-B51 status and the result of the pathergy test were recorded from the charts. An ophthalmological evaluation was performed if necessary.

The skin lesions were scored as 0 : absent, 1 : $1-5,2: 6-10,3: 11-15,4: 16-20$, and 5: >20 in 
Table 1 Demographic features and the frequency and regional distribution of skin lesions in study groups. Results are given as mean (SD)

\begin{tabular}{|c|c|c|c|c|c|}
\hline & $\begin{array}{l}B S^{*} \text { and arthritis } \\
(n=44)\end{array}$ & $\begin{array}{l}\text { BS without } \\
\text { arthritis }(n=42)\end{array}$ & $\begin{array}{l}\text { Rheumatoid } \\
\text { arthritis }(n=21)\end{array}$ & $\begin{array}{l}\text { Healthy controls } \\
(n=33)\end{array}$ & $p$ Value \\
\hline Male/female (n) & $32 / 12$ & $31 / 11$ & $5 / 16$ & $28 / 5$ & \\
\hline Age (years) & $37.8(8.9)$ & $35.5(6.4)$ & $48.8(14)$ & $40.1(8.1)$ & $<0.05$ \\
\hline Disease duration (years) & $5.6(1.6)$ & $4.7(1.2)$ & $3.2(2.1)$ & - & NS \\
\hline \multicolumn{6}{|l|}{ Overall } \\
\hline Comedone & $1.11(1.4)$ & $1.26(1.8)$ & $1.14(1.7)$ & $0.97(1.5)$ & NS \\
\hline Papule & $1.27(1.3)$ & $0.6(0.8)$ & $0.7(1.6)$ & $0.91(1.1)$ & 0.0037 \\
\hline Pustule & $1.3(1.3)$ & $0.48(0.6)$ & $0.14(0.4)$ & $0.12(0.4)$ & $<0.0001$ \\
\hline \multicolumn{6}{|l|}{ Face } \\
\hline Comedone & $0.91(1.1)$ & $1.06(1.7)$ & $1.14(1.7)$ & $0.7(1.4)$ & NS \\
\hline Papule & $0.98(1.3)$ & $0.1(0.3)$ & $0.33(0.9)$ & $0.06(0.2)$ & 0.0001 \\
\hline Pustule & $0.45(0.9)$ & $0.19(0.5)$ & $0.05(0.2)$ & $0.01(0.1)$ & 0.0004 \\
\hline \multicolumn{6}{|l|}{ Back } \\
\hline Comedone & $0.20(0.5)$ & $0.21(0.5)$ & $0.01(0.1)$ & $0.33(0.9)$ & NS \\
\hline Papule & $0.98(1.3)$ & $0.45(0.7)$ & $0.33(1.1)$ & $0.15(1.1)$ & 0.0038 \\
\hline Pustule & $0.95(1.2)$ & $0.19(0.4)$ & $0.1(0.3)$ & $0.12(0.4)$ & $<0.0001$ \\
\hline \multicolumn{6}{|l|}{ Chest } \\
\hline Comedone & $0.23(0.8)$ & $0.14(0.8)$ & $0.01(0.1)$ & $0.21(0.9)$ & NS \\
\hline Papule & $0.32(0.7)$ & $0.12(0.3)$ & $0.01(0.1)$ & $0.06(0.2)$ & 0.0067 \\
\hline Pustule & $0.43(0.9)$ & $0.17(0.4)$ & $0.01(0.1)$ & $0.01(0.1)$ & 0.0002 \\
\hline
\end{tabular}

${ }^{\star}$ BS $=$ Behçet's syndrome.

tp Values indicate the difference among study groups: $p<0.05$ indicates that the group with the highest value had a significantly increased number of lesions compared with the other groups. (Kruskal-Wallis-one way analysis of variance).

number. A Kruskal-Wallis one way analysis of variance test was used to compare data between the groups. The presence or absence of clinical manifestations as listed above and the results of the HLA-B51 and pathergy tests were compared for the two groups with BS by the $\chi^{2}$ test.

\section{Results}

Patients with RA were predominantly female and about a decade older than those in other groups $(p<0.05)$ (table 1$)$. The occurrence of clinical features other than the papulopustular lesions was similar in those patients with BS with and without arthritis (fig 2). Both groups with BS were also similar in age, sex, disease duration, and drugs used.

Although the prevalence of comedones was not significantly different between the four groups, the numbers of papules $(p<0.004)$ and pustules $(p<0.0001)$ were higher in patients with BS with arthritis than in the other three groups (table 1).

Eight patients in the group with BS with arthritis and nine in the group without arthritis had eye disease. Papulopustular lesions were not increased in patients with BS with eye involvement (data not given).

Of the 44 patients with BS with arthritis, 12 had one, 30 had one to four, and two had more

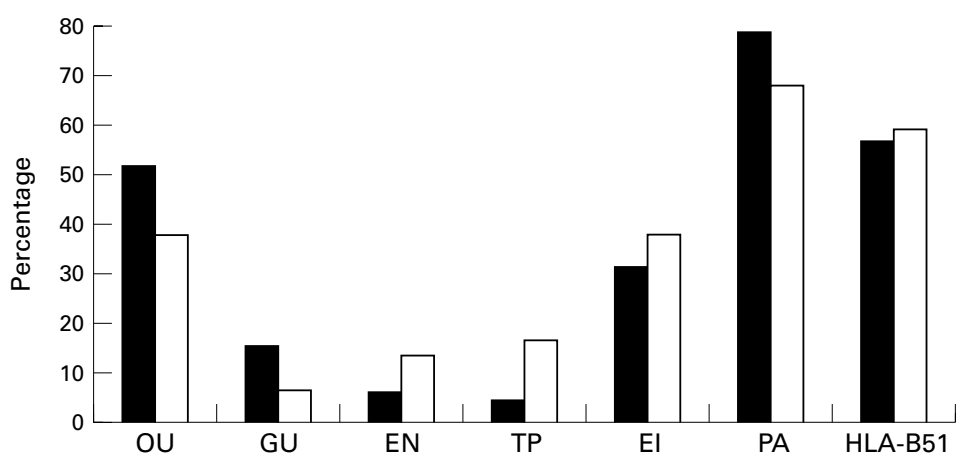

Figure 2 The prevalence of clinical features other than the acneiform skin lesion in the patients with Behçet's syndrome with (solid bars) and without arthritis (open bars). $O U=$ oral ulcers; $G U=$ genital ulcers; $E N=$ erythema nodosum; $T P=$ thrombophlebitis; $E I=$ eye involvement; $P A=$ pathergy test. than four joints affected. The knee was the joint most commonly affected (23 patients), followed by the ankle in 12 patients. There were no patients with inflammatory low back pain, heel pain, or distal interphalangeal joint disease. Arthritis was among the presenting manifestations in eight patients with newly diagnosed BS. The number of diseased joints did not affect the prevalence of skin lesions (data not given). Twelve patients in the group with BS with arthritis and seven in the group without arthritis had low back pain. However, an $x$ ray examination of their lumbosacral and sacroiliac joints showed no evidence of lumbar spondyloarthropathy or sacroiliitis.

\section{Discussion}

The results support our hypothesis that skin papules and pustules are associated with arthritis in BS.

The prevalence of other disease manifestations was similar in patients with BS with or without arthritis. Furthermore, the drugs used at the time of study were similar in both groups, suggesting that disease severity was also similar in both groups. On the other hand, the probability remains that both the presence of arthritis and the pustular lesions simply reflected more severe disease within the organs affected. Our clinical impression was that this was not the case. However, a formal assessment of disease severity in the two groups was not made and we must add that currently there is no widely accepted severity index available for BS.

The patients with RA in this study were examined so that the prevalence of papulopustular lesions in patients with BS could be compared with that in another group of patients with a chronic inflammatory condition. However, the prevalence of acne, in general, decreases with age ${ }^{5}$ and our patients with RA were somewhat older than the patients with BS, even though they were well beyond early adulthood when acne lesions are most common. In retrospect, it would have been preferable to have had a control group strictly 
comparable in age and sex. Furthermore, in such studies in the future the inclusion of control groups with psoriasis or SAPHO, or both, would also be desirable, especially in the light of the following discussion.

The association of arthritis and papulopustular lesions has been well described in both SAPHO syndrome and psoriatic arthritis. In the pathogenesis of psoriatic arthritis streptococcus species $^{67}$ and in SAPHO syndrome Propionibacterium acnes ${ }^{8}$ have been implicated. Edlund et al carried out an open biopsy study of affected joints and para-articular bone and found that in seven of 15 patients with palmoplantar pustulosis and sternocostoclavicular arthro-osteitis, Propionibacterium acnes was grown in least two of five cultures. ${ }^{9}$ The role of micro-organisms in the pathogenesis of BS has also been studied. Lehner et al analysed serum samples from patients with BS by immunoblot assay and found that IgA antibodies to the recombinant $65 \mathrm{kDa}$ mycobacterial heat shock protein and to soluble protein extracts of Streptococcus sanguis, ST3, KTH-1, KTH-2, and KTH-3, were significantly increased. ${ }^{10}$ A Japanese group reported that patients with BS showed significantly higher antibody titres to Streptococcus sanguis strains 113-20, 114-23, and 118-1 than control groups. ${ }^{11}$ Finally, Çalgüneri et al found that prophylactic penicillin treatment significantly reduced the number of arthritis episodes. ${ }^{12}$ Taken together, these findings suggest an infectious cause in the pathogenesis of BS, akin to a "reactive" arthritis. However, unlike the reactive arthritides, enthesitis and the HLA-B27 association are absent in BS. The nature of the genital lesions is quite different and, as was true for our patients, sacroiliitis is usually not present in BS.

The association of arthritis with acne and not with the other manifestations also raises the intriguing possibility that more than one pathogenetic mechanism might be operative in BS. This contention is also supported by the observation that one agent, thalidomide, is uniformly effective for almost all the skin mucosa manifestations of BS except the erythema nodosum lesions, which it actually exacerbates. ${ }^{13}$

1 Ergun T, Gürbüz O, Dogusoy G, Mat C, Yazici $H$. Histopathologic features of the spontaneous pustular lesions of Behçet's syndrome. Int J Dermatol 1998;37:194-6.

2 Kahn MF, Chamot AM. SAPHO syndrome. Rheum Dis Clin North Am 1992;18:225-46.

3 Criteria for diagnosis of Behçet's disease. International Study Group for Behçet's Disease. Lancet 1990;335:107880

4 Arnett FC, Edworthy SM, Bloch DA, McShane DJ, Fries JF, Cooper NS, et al. The American Rheumatism Association 1987 revised criteria for the classification of rheumatoid arthritis. Arthritis Rheum 1988;31:315-24.

5 Cunliffe WJ. Natural history of acne. In: Cunliffe WJ, ed. Acne. London: Martin Dunitz, 1989:2-10.1.

6 Sieper J, Braun J. Pathogenesis of spondylarthopathies: persistent bacterial antigen, autoimmunity, or both? Arthritis Rheum 1995;38:1547-54.

7 Grinlinton FM, Skinner MA, Birchall NM, Tan PLJ. Gamma-delta $^{+} \mathrm{T}$ cells from patients with psoriatic and rheumatoid arthritis respond to streptococcal antigen. J Rheumatol 1993;20:983-7.

8 Boutin RD, Resnick D. The SAPHO syndrome: an evolving concept for unifying several idiopathic disorders of bone and skin. AJR 1998;170:585-91.

9 Edlund E, Johnsson U, Lidgren L, Pettersson H, Sturfelt G, Svensson B, et al. Palmoplantar pustulosis and sternocostoSvensson B, et al. Palmoplantar pustulosis and sternocosto-
clavicular arthro-arthritis. Ann Rheum Dis 1988;47:80915.

10 Lehner T, Lavery E, Smith R, van der Zee R, Mizushima Y, Shinnick T. Association between the 65-kilodalton heat shock protein, Streptococcus sanguis, and the corresponding antibodies in Behçet's syndrome. Infect Immun 1991;59:1434-41.

11 Yokota K, Hayashi S, Fujii N, Yoshikawa K, Kotake S, Isogai E, et al. Antibody response to oral streptococci in
Behçet's disease. Microbiol Immunol 1992;36:815-22.

12 Çalgüneri M, Kiraz S, Ertenli I, Benekli M, Karaarslan Y, Celik I. The effect of prophylactic penicillin treatment on the course of arthritis episodes in patients with Behçet's disease. Arthritis Rheum 1996;39:2062-5.

13 Hamuryudan V, Mat C, Saip S, Özyazgan Y, Siva A, Yurdakul S, et al. Thalidomide in the treatment of the mucocutaneus lesions of the Behçet's syndrome. Ann Intern Med 1998;128:443-50. 